\title{
Interpretación de la cápsula endoscópica: el papel del personal no especializado
}

\author{
I. Fernández-Urién, E. Espinet, N. Pérez, M. Betés, M. Herráiz, C. Carretero y M. Muñoz-Navas \\ Servicio de Aparato Digestivo. Clínica Universitaria de Navarra. Pamplona
}

\section{RESUMEN}

Introducción y objetivos: la cápsula endoscópica (CE) ha supuesto una nueva era en el estudio del intestino delgado. No obstante, el tiempo empleado por el gastroenterólogo en este procedimiento es mayor del deseable y no se han evaluado completamente alternativas al personal especializado. El objetivo de este estudio es evaluar la precisión de personal no especializado en la interpretación de la CE.

Material y métodos: un gastroenterólogo con experiencia en CE y personal no especializado revisaron independientemente 20 procedimientos. Los hallazgos de cada participante eran desconocidos por el resto. Un consenso formado por los participantes y un segundo gastroenterólogo fue empleado como gold standard. Se analizaron número, tipo y localización de las imágenes seleccionadas y tiempo de evacuación gástrica (tEG), tiempo de tránsito en intestino delgado (tTID) y tiempo empleado por los participantes.

Resultados: la sensibilidad y la especificidad global fueron del 79 y $99 \%$ para el gastroenterólogo; del 86 y $43 \%$ para la enfermera; y del 80 y $57 \%$ para el residente. Las 34 lesiones "mayores" consideradas por consenso fueron detectadas por los tres participantes. El acuerdo entre consenso y participantes para clasificar e interpretar las imágenes fue de buena a excelente ( $\kappa$ de 0,55 a 1). No se encontraron diferencias estadísticamente significativas en el tEG y tTID obtenido por consenso y participantes. El gastroenterólogo fue el más rápido en revisar los procedimientos $(51,9 \pm 13,5$ minutos versus $62,2 \pm 19$ y $60,9 \pm 17,1$ para enfermera y residente, respectivamente; $\mathrm{p}<0,05)$.

Conclusiones: el personal no especializado podría ser el complemento perfecto al gastroenterólogo en la interpretación de la CE, aunque este debería supervisar sus hallazgos. Los beneficios de esta alternativa deberían ser contrastados en el futuro por análisis coste-efectividad.

Palabras clave: Cápsula endoscópica. Enfermera. Residente. Personal no especializado.

\begin{abstract}
Background and aims: capsule endoscopy (CE) allows for a new era in small-bowel examination. Nevertheless, physicians' time for CE-interpretation remains longer than desirable. Alternative strategies to physicians have not been widely investigated. The aim of this study was to evaluate the accuracy of physician extenders in CE-interpretation.

Material and methods: one CE-experienced gastroenterologist and two physician extenders reviewed independently $20 \mathrm{CE}-$ procedures. Each reader was blinded to the findings of their colleagues. A consensus formed by the readers and a second CE-experienced gastroenterologist was used as gold standard. Number, type and location of images selected, character of CEexams and their relationship with indications were recorded. Gastric emptying time (GEt), small-bowel transit time (SBTt) and time spent by readers were also noted.
\end{abstract}

Results: sensitivity and specificity for "overall" lesions was 79 and $99 \%$ for the gastroenterologist; 86 and $43 \%$ for the nurse; and 80 and $57 \%$ for the resident. All 34 "major" lesions considered by consensus were found by the readers. Agreement between consensus and readers for images classification and procedures interpretation was good to excellent ( $\kappa$ from 0.55 to 1 ). No significant differences were found in the GEt and SBTt obtained by consensus and readers. The gastroenterologist was faster than physician extenders (mean time spent was $51.9 \pm 13.5$ minutes versus $62.2 \pm 19$ and $60.9 \pm 17.1$ for nurse and resident, respectively; $p<0.05$ )

Conclusions: physician extenders could be the perfect complement to gastroenterologists for CE-interpretation but gastroenterologists should supervise their findings. Future cost-efficacy analyses are required to assess the benefits of this alternative.

Key words: Capsule endoscopy. Nurse. Resident. Physician extenders.

Fernández-Urién I, Espinet E, Pérez N, Betés M, Herráiz M, Carretero C, Muñoz-Navas M. Interpretación de la cápsula endoscópica: el papel del personal no especializado. Rev Esp Enferm Dig 2008; 100: 219-224. 


\section{INTRODUCCIÓN}

La cápsula endoscópica (CE) supone una nueva era en el estudio del intestino delgado. Como demuestran recientes estudios, la CE es superior al tránsito baritado, al entero-TC y a la enteroscopia convencional (1-10). Actualmente, la interpretación de la CE está limitada al gastroenterólogo y el tiempo que emplea en cada procedimiento es variable. Parece depender de la experiencia y concentración del explorador, así como del número de lesiones detectadas. Habitualmente, consume alrededor de 1 hora (rango 30-120 minutos) según las series publicadas $(1-3,6,9)$, lo que resulta más de lo deseable. El papel de personal no especializado en la interpretación de la $\mathrm{CE}$ no está del todo estudiado y podría ser beneficioso. Nuestra hipótesis es que personal no especializado, debidamente entrenado, podría evaluar en primer lugar los procedimientos mientras que los gastroenterólogos podrían revisar únicamente las imágenes seleccionadas. Significaría un ahorro de tiempo y probablemente de dinero. Los objetivos de este estudio fueron evaluar la precisión diagnóstica y el tiempo empleado por personal especializado y no especializado en la revisión e interpretación de la CE.

\section{MATERIAL Y MÉTODOS}

\section{Pacientes y procedimientos}

Un total de 20 procedimientos fueron incluidos en el estudio. Todos fueron realizados con la PillCam ${ }^{\mathrm{TM}} \mathrm{SB}$ (Given Imaging Ltd., Yoqneam, Israel) y todos los pacientes ayunaron desde la noche anterior al procedimiento. No se utilizaron laxantes o procinéticos en los procedimientos.

\section{Participantes y entrenamiento}

Los procedimientos fueron revisados independientemente, a una velocidad no establecida, por un gastroenterólogo con experiencia en CE y personal no especializado: un residente de digestivo y una enfermera. Los tres participantes recibieron los mismos datos clínicos y desconocían los hallazgos del resto. En el momento del estudio, el gastroenterólogo tenía una experiencia en CE de más de 150 procedimientos. Ni enfermera ni residente tenían experiencia en CE aunque sí en imágenes del intestino delgado. En estos momentos no hay descritas medidas específicas para el entrenamiento en CE y por ello se emplearon con el personal no especializado las medidas sugeridas por ASGE (11). Además, completaron un curso de entrenamiento en CE en el que se enfatizó en el manejo del hardware y software del sistema, así como en el reconocimiento y caracterización de las lesiones. Antes del comienzo del estudio, enfermera y residente revisaron 15 procedimientos bajo la tutela de un gastroenterólogo con experiencia en $\mathrm{CE}$, quien finalmente aseguró su compe- tencia. Adicionalmente y durante los procedimientos, se permitió el uso de la web de Given Imaging (www.givenimaging.com) y el Atlas de Cápsula Endoscópica (12).

\section{Variables}

Las variables incluidas en el estudio y los criterios empleados para su clasificación, se muestran en las tablas I y II (13). Todas las imágenes sospechosas fueron seleccionadas y clasificadas por los participantes de acuerdo a su tipo y localización. Los participantes además clasificaron los procedimientos de acuerdo a su carácter y anotaron la relación entre la indicación del procedimiento y los hallazgos. El tiempo de evacuación gástrica (tEG) y de tránsito en intestino delgado (tTID), así como el tiempo empleado por cada participante (tR), fueron también anotados. Todos los participantes estaban familiarizados con estas variables desde el periodo de entrenamiento.

Tabla I. Variables derivadas de las imágenes seleccionadas y criterios para su clasificación

\begin{tabular}{|c|c|}
\hline Número & Número de imágenes seleccionadas por cada participante \\
\hline \multicolumn{2}{|l|}{ Tipo* } \\
\hline Vasculares & $\begin{array}{l}\text { Definidas como aquellas que aparecen como puntos rojos, pequeñas, } \\
\text { planas y a veces en forma de araña }\end{array}$ \\
\hline Inflamatorias & $\begin{array}{l}\text { Definidas como aquellas que muestran un engrosamiento de pliegues } \\
\text { con edema, eritema o nodularidad }\end{array}$ \\
\hline Erosión-úlcera & $\begin{array}{l}\text { Definidas como aquellas que aparecen con pérdida de mucosa superficial } \\
\text { localizada y generalmente asociadas a eritema y/o irregularidad de la mu- } \\
\text { cosa circundante }\end{array}$ \\
\hline Pólipo-neoplasia & $\begin{array}{l}\text { Definidas como aquellas que aparecen como masa polipoidea, vegetante } \\
\text { o submucosa, ulcerada o no y con mucosa circundante normal }\end{array}$ \\
\hline Sangrado activo & $\begin{array}{l}\text { Definidas como aquellas que aparecen como sangrado activo, con/sin } \\
\text { fuente apreciable }\end{array}$ \\
\hline Mínimas & Definidas como aquellas, a pesar de su tipo, inferiores a 4 mm (en pantalla) \\
\hline Otras & $\begin{array}{l}\text { Definidas como resto de lesiones encontradas (patrón en mosaico, xanto- } \\
\text { mas, hiperplasia linfoide,...) }\end{array}$ \\
\hline \multicolumn{2}{|l|}{ Localización } \\
\hline Duodeno & $\begin{array}{l}\text { Mucosa entre píloro y ligamento de Treitz (cuadrante superior derecho } \\
\text { del localizador). Tránsito corto y rápido (habitualmente < } 15 \text { min). Otros } \\
\text { criterios para la localización duodenal fueron el flujo biliar, la visualización } \\
\text { de la papila, patrón vellositario evidente, ausencia de vascularización visi- } \\
\text { ble y presencia de linfangiectasia }\end{array}$ \\
\hline Yeyuno & $\begin{array}{l}\text { Mucosa entre el ligamento de Treitz e íleon (ver íleon), lejos de la primera } \\
\text { imagen duodenal y cecal. Tránsito largo y lento (habitualmente > } 60 \\
\text { min). Otros criterios: patrón vellositario no llamativo, patrón vascular lla- } \\
\text { mativo, ausencia de bilis y peristalsis evidente }\end{array}$ \\
\hline Íleon & $\begin{array}{l}\text { Mucosa entre yeyuno (ver yeyuno) y primera imagen cecal. Tránsito corto } \\
\text { y lento (habitualmente > } 30 \text { min). Otros criterios: patrón vellositario no } \\
\text { evidente, patrón vascular evidente (vasos finos), restos fecales frecuentes, } \\
\text { peristalsis muy llamativa e imágenes típicas como hiperplasia linfoide }\end{array}$ \\
\hline Otras & Imágenes localizadas en el esófago, estómago y colon \\
\hline \multicolumn{2}{|l|}{ Intensidad } \\
\hline Mayores & Definidas como aquellas $>7 \mathrm{~mm} * *$, sangrado activo o lesiones difusas \\
\hline No mínimas & Definidas como aquellas que miden $4-7^{* *} \mathrm{~mm}$ \\
\hline Mínimas & Definidas como aquellas, a pesar de su tipo, $<4$ mm $* \star$ \\
\hline
\end{tabular}

*Modificado de CE-Minimal Standard Terminology (CE-MST) (13); **Tamaño medido en pantalla. 
Tabla II. Variables derivadas de los procedimientos y criterios para su clasificación

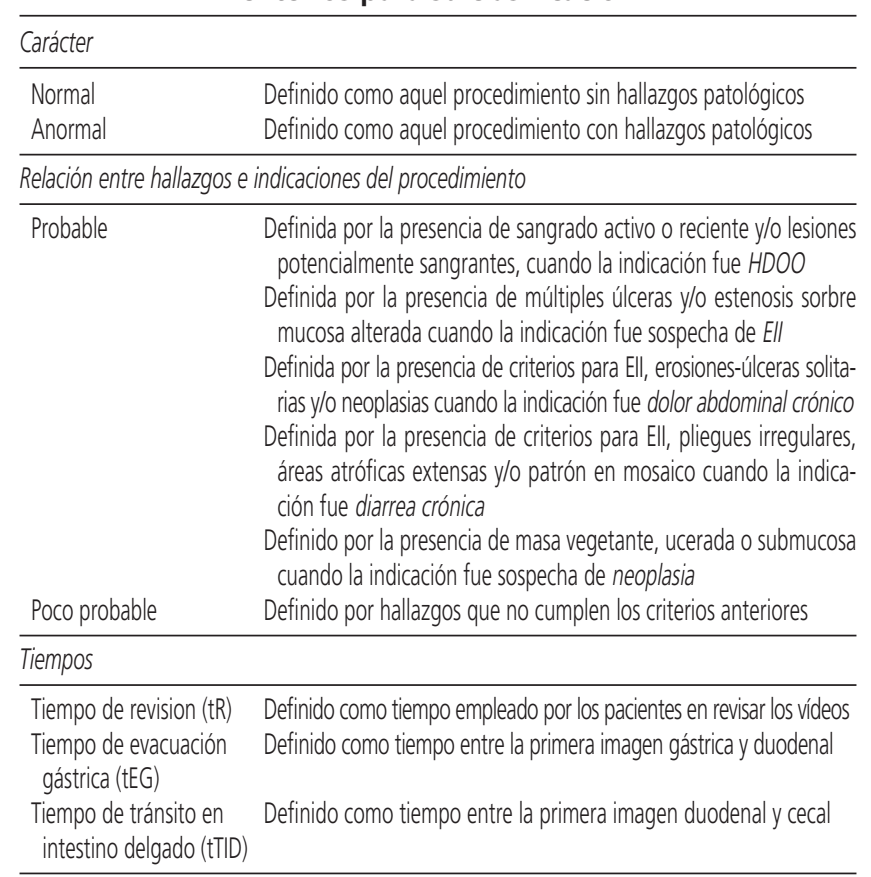

\section{Gold standard y análisis estadístico}

En ausencia de un gold standard para comparaciones, se creó un consenso formado por los participantes y un segundo gastroenterólogo con experiencia en CE. El consenso revisó cada una de las imágenes seleccionadas por los participantes. Entonces se discutía si eran patológicas y además su tipo, localización e intensidad. De igual manera se realizó con las variables relacionadas con los procedimientos. La opinión del consenso junto con los datos clínico-analíticos fue considerada como gold standard, analizando la precisión diagnóstica de los participantes. La selección de imágenes y la caracterización de imágenes y procedimientos, así como los tiempos obtenidos por los participantes, fueron comparados mediante ANOVA con comparaciones post-hoc (test de Dunnett) cuando fue necesario. Una $p<0,05$ fue considerada estadísticamente significativa. El acuerdo consenso-participantes para imágenes y procedimientos fue evaluado mediante el índice kappa $(\kappa)$, tomándose en cuenta los valores de $\kappa$ sugeridos por Fleiss $(14)(<0,40$ acuerdo pobre; 0,40-0,75 acuerdo bueno; > 0,75 excelente acuerdo). Los datos del ANOVA se muestran como media \pm desviación estándar (DS) y los valores de $\kappa$ son presentados con su intervalo de confianza al 95\% (IC 95\%). El análisis estadístico fue realizado con la versión 12.0 del SPSS para Windows (SPSS Inc. Chicago, Illinois, EE.UU.) y el SISA online statistical analysis (http://home.clara.net/sisa/index.htm).

\section{RESULTADOS}

\section{Selección de imágenes}

Las imágenes consideradas patológicas por consenso y participantes y su caracterización, así como los resultados del ANOVA-Dunnett, se muestran en la tabla III. Los valores de $\kappa$ para el acuerdo consenso-participantes se muestran en la tabla IV.

Tabla III. Selección de imágenes y clasificación

\begin{tabular}{|c|c|c|c|c|c|}
\hline \multicolumn{6}{|c|}{ N. ${ }^{\circ}$ de imágenes seleccionadas/X (DS) } \\
\hline & C & G & E & $R$ & Sig. \\
\hline \multicolumn{6}{|l|}{$\begin{array}{l}\text { Global } \\
\text { Intensidad }\end{array}$} \\
\hline Lesiones "mayores" & $32 / 1,60(1,78)$ & 11601 & $32 / 1,60(1,78)$ & $32 / 1,6$ & J \\
\hline Lesiones "no-mínimas" & $174 / 8,70(5,33)$ & $161 / 8,0$ & $169 / 8,45(5,02)$ & $158 / 7,90(4,95)$ & 0,326 \\
\hline Lesiones "mínimas" & $94 / 4,70(3,75)$ & $44 / 2,20(2,76)^{*}$ & $106 / 5,30(3,72)$ & $87 / 4,35(2,96)$ & $<0,001$ \\
\hline \multicolumn{6}{|c|}{ 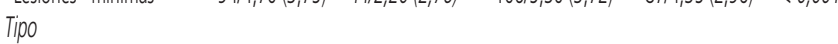 } \\
\hline Vasculares & $54 / 2,70(2,83)$ & $52 / 2,60$ & $51 / 2,55(2,72)$ & $47 / 2,3$ & 0,298 \\
\hline Inflamatorias & 100 (100) & $34 / 1,70$ & $44 / 2,20(2,19)$ & $40 / 2$ & 0,145 \\
\hline Erosión-úlcera & $41 / 2,05(2,32)$ & $41 / 2,05(2,48)$ & $34 / 1,70(1,89)$ & $39 / 1,95(1,84)$ & 0,447 \\
\hline Pólipo-neoplasia & $14 / 0,70(1,26)$ & $15 / 0,75(1,25)$ & $15 / 0,75(1,40)$ & $13 / 0,65(1,22)$ & 0,871 \\
\hline Sangrado activo & $4 / 0,20(0,52)$ & $4 / 0,20(0,52)$ & $4 / 0,20(0,52)$ & $4 / 0,20(0,52)$ & $\S$ \\
\hline Mínimas & $94 / 4,70(3,75)$ & $44 / 2,20(2,76)^{*}$ & $106 / 5,30(3,72)$ & $87 / 4,35(3,01)$ & $<0,00$ \\
\hline Otras & $53 / 2,65(2,15)$ & $47 / 2,35(1,95)$ & $53 / 2,65(2,13)$ & $47 / 2,35(1,89)$ & 0,24 \\
\hline \multicolumn{6}{|l|}{ Localización } \\
\hline Duodeno & $47 / 2,35$ (1 & $38 / 1,90$ & $54 / 2,70(1,80)$ & $51 / 2$ & 0,054 \\
\hline Yeyuno & $139 / 6,95(4,35)$ & $113 / 5,65(3,85)^{*}$ & $155 / 7,75(4,75)$ & $141 / 7,05(4,33)$ & 0,016 \\
\hline Illeon & $85 / 4,25(4,16)$ & $61 / 3,05(3,50)$ & $71 / 3,55(2,89)$ & $61 / 3,05(3,23)$ & 0,144 \\
\hline Otras & $29 / 1,45(1,23)$ & $25 / 1,25(1,11)$ & $27 / 1,35(1,04)$ & $24 / 1,20(0,89)$ & 0,103 \\
\hline
\end{tabular}

C: consenso; G: gastroenterólogo; E: enfermera; R: residente; Sig.: significación de ANOVA (comparación de medias); *Diferencias estadísticamente significativas entre consenso y participante (el test de Dunett fue realizado si sig. $<0,05$ ); $\S$ : acuerdo total (varianza $=0$ )

Tabla IV. Acuerdo interobservador

\begin{tabular}{|c|c|c|c|}
\hline \multicolumn{4}{|c|}{ Valores de к (IC 95\%) } \\
\hline & $C-G$ & $C-E$ & $C-R$ \\
\hline Global & $0,61(0,53-0,70)$ & $0,30(0,18-0,40)$ & $0,33(0,22-0,43)$ \\
\hline \multicolumn{4}{|l|}{ Intensidad } \\
\hline Lesiones "mayores" & 1 & 1 & 1 \\
\hline Lesiones "no mínimas" & $0,73(0,60-0,86)$ & $0,51(0,34-0,68)$ & $0,18(0,01-0,34)$ \\
\hline Lesiones "mínimas" & $0,31(0,13-0,48)$ & $0,05(0-0,21)$ & $0,30(0,14-0,45)$ \\
\hline \multicolumn{4}{|l|}{ Tipo } \\
\hline Global & $0,79(0,74-0,82)$ & $0,64(0,59-0,69)$ & $0,64(0,59-0,69)$ \\
\hline Vasculares & $0,98(0,95-1)$ & $0,85(0,78-0,93)$ & $0,85(0,77-0,93)$ \\
\hline Inflamatorias & $0,91(0,83-0,98)$ & $0,78(0,68-0,88)$ & $0,75(0,63-0,86)$ \\
\hline Erosión-úlcera & $0,94(0,89-0,99)$ & $0,77(0,67-0,88)$ & $0,77(0,67-0,88)$ \\
\hline Pólipo-neoplasia & $0,96(0,89-1)$ & $0,82(0,66-0,97)$ & $0,81(0,64-0,97)$ \\
\hline Sangrado activo & 1 & 1 & 1 \\
\hline Mínimas & $0,57(0,46-0,67)$ & $0,48(0,38-0,58)$ & $0,59(0,49-0,68)$ \\
\hline Otras & $0,91(0,86-0,97)$ & $0,89(0,82-0,95)$ & $0,79(0,70-0,88)$ \\
\hline \multicolumn{4}{|l|}{ Localización } \\
\hline Global & $0,76(0,71-0,81)$ & $0,57(0,51-0,63)$ & $0,55(0,49-0,61)$ \\
\hline Duodeno & $0,88(0,80-0,95)$ & $0,67(0,56-0,78)$ & $0,67(0,56-0,78)$ \\
\hline Yeyuno & $0,77(0,71-0,84)$ & $0,58(0,50-0,66)$ & $0,56(0,47-0,65)$ \\
\hline Íleon & $0,80(0,72-0,87)$ & $0,60(0,50-0,70)$ & $0,61(0,51-0,71)$ \\
\hline Otras & $0,92(0,84-1)$ & $0,96(0,90-1)$ & $0,81(0,70-0,93)$ \\
\hline
\end{tabular}

C: consenso; G: gastroenterólogo; E: enfermera; R: residente; IC 95\%: intervalo de confianza al $95 \%$; Valores de corte para $\kappa(14):<0,40$ pobre; $0,40-0,75$ bueno: $>0,75$ excelente. 


\section{Número de imágenes}

Se observaron diferencias estadísticamente significativas en el número "global" de imágenes consideradas patológicas por consenso y participantes ( $p<0,001)$. El test de Dunnett mostró que el gastroenterólogo fue el único que seleccionó menos imágenes que el consenso $(\mathrm{p}<0,05)$. No obstante, existió un nivel de acuerdo consenso-gastroenteró$\operatorname{logo}$ bueno $(\kappa=0,61)$ y consenso-personal no especializado pobre $(\kappa=0,30$ y 0,33 para enfermera y residente, respectivamente). Cuando las lesiones fueron clasificadas por intensidad, se observaron únicamente diferencias en el número de lesiones "mínimas" seleccionadas por consenso y participantes ( $\mathrm{p}<0,001)$. El gastroenterólogo fue de nuevo el único que seleccionó menos lesiones mínimas que el consenso $(\mathrm{p}<0,05)$. Además, se observó un pobre acuerdo consensoparticipantes para estas lesiones ( $\kappa$ entre 0,05 y 0,31 ). Por otro lado, no existieron diferencias en el número de lesiones "mayores" o "no-mínimas" seleccionadas por consenso y participantes. Existió un acuerdo absoluto en la detección de lesiones "mayores" $(\kappa=1)$ y de bueno a pobre para lesiones "no-mínimas" $(\kappa=0,73$ consenso-gastroenterólogo; $\kappa=$ 0,51 consenso-enfermera; у $\kappa=0,18$ consenso-residente).

\section{Tipo y localización de imágenes}

No se observaron diferencias significativas entre consenso y participantes en el número de imágenes seleccionadas de acuerdo a su tipo y localización, excepto para las lesiones tipo "mínimas" ( $\mathrm{p}<0,001)$ y las localizadas en yeyuno $(\mathrm{p}<0,05)$. Estas diferencias se debieron a la selección del gastroenterólogo como demostraron las comparaciones post-hoc, ya que seleccionó menos lesiones "mínimas" y menos lesiones localizadas en yeyuno que el consenso $(p<0,05)$. El acuerdo global consenso-participantes para la clasificación de las lesiones en función del tipo fue excelente para el gastroenterólogo $(\kappa=0,79)$ y bueno para enfermera y residente ( $\kappa=0,64$, para ambos). En la tipificación de lesiones, los participantes obtuvieron valores de $\kappa$ superiores a 0,75 (acuerdo excelente), excepto para las lesiones "mínimas" ( $\kappa$ entre 0,48-0,59). El acuerdo global consenso-gastroenterólogo para la clasificación de las lesiones en función de la localización fue excelente $(\kappa=0,76)$ y buena para enfermera y residente $(\kappa=0,57$ y $\kappa=0,55$, respectivamente). Para cada localización, el acuerdo consenso-gastroenterólogo fue excelente en todas las opciones ( $\kappa$ entre 0,77 y 0,92 ), excelente para enfermera y residente en "otras" localizaciones $(\kappa=0,89$ y $\kappa=$ 0,79 , respectivamente) y buena para la localización en duodeno, yeyuno e íleon ( $\kappa$ entre 0,56 y 0,69 ).

\section{Precisión diagnóstica}

La sensibilidad para el "global" de las lesiones fue superior en personal no especializado ( 86 y $80 \%$ para en- fermera y residente versus $79 \%$ para el gastroenterólogo) pero la especificidad fue inferior (99\% para el gastroenterólogo versus 43 y $57 \%$ para enfermera y residente). Como se ha comentado anteriormente, todos los participantes seleccionaron las 34 lesiones "mayores" consideradas por consenso, resultando en una sensibilidad y especificidad de 1. Para lesiones "no-mínimas", la sensibilidad de cada participante fue superior al $80 \%$ y la especificidad del gastroenterólogo fue de nuevo superior que la de enfermera y residente (96 versus 59 y $41 \%$, respectivamente). La sensibilidad para lesiones "mínimas" fue pobre en general pero superior para enfermera y residente (67 y $69 \%$, respectivamente versus $42 \%$ para el gastroenterólogo) y la especificidad fue del 1 para el gastroenterólogo, del $39 \%$ para la enfermera y $61 \%$ para el residente (más detalles en la tabla V).

Tabla V. Precisión diagnóstica para la detección de lesiones

\begin{tabular}{|c|c|c|c|c|c|}
\hline & $S(\mid 1 C 95 \%)$ & $E(\mid C 95 \%)$ & J & $R P+(\mid C 95 \%)$ & $R P-(\mid C 95 \%)$ \\
\hline \multicolumn{6}{|c|}{ Global } \\
\hline $\begin{array}{l}G \\
E \\
R\end{array}$ & $\begin{array}{l}0,79(0,74-0,83) \\
0,86(0,82-0,90) \\
0,80(0,75-0,84)\end{array}$ & $\begin{array}{l}0,99(0,97-1,01) \\
0,43(0,33-0,54) \\
0,57(0,46-0,67)\end{array}$ & $\begin{array}{l}0,78 \\
0,29 \\
0,37\end{array}$ & $\begin{array}{c}69,23(9,85-486,44) \\
1,51(1,25-1,82) \\
1,84(1,44-2,36)\end{array}$ & $\begin{array}{l}0,22(0,17-0,27) \\
0,33(0,23-0,48) \\
0,36(0,27-0,48)\end{array}$ \\
\hline \multicolumn{6}{|c|}{ Lesiones "mayores" } \\
\hline $\begin{array}{l}G \\
E \\
R\end{array}$ & $\begin{array}{l}1 \\
1 \\
1\end{array}$ & $\begin{array}{l}1 \\
1 \\
1\end{array}$ & $\begin{array}{l}1 \\
1 \\
1\end{array}$ & $\begin{array}{l}\infty \\
\infty \\
\infty\end{array}$ & $\begin{array}{l}0 \\
0 \\
0\end{array}$ \\
\hline
\end{tabular}

Lesiones "no minimas"

\begin{tabular}{lccccc}
\hline$G$ & $0,92(0,88-0,96)$ & $0,96(0,89-1)$ & 0,88 & $24,83(3,63-170)$ & $0,08(0,05-0,14)$ \\
$E$ & $0,93(0,89-0,97)$ & $0,59(0,41-0,78)$ & 0,52 & $2,29(1,45-3,61)$ & $0,12(0,06-0,22)$ \\
$R$ & $0,82(0,76-0,87)$ & $0,41(0,22-0,59)$ & 0,23 & $1,38(1-1,90)$ & $0,45(0,26-0,78)$ \\
\hline Lesiones "minimas" & & & & \\
\hline$G$ & $0,42(0,32-0,51)$ & 1 & 0,42 & $\infty$ & $0,58(0,50-0,69)$ \\
$E$ & $0,67(0,58-0,77)$ & $0,39(0,27-0,51)$ & 0,06 & $1,09(0,86-1,39)$ & $0,85(0,56-1,30)$ \\
$R$ & $0,69(0,60-0,78)$ & $0,61(0,49-0,73)$ & 0,30 & $1,79(1,27-2,51)$ & $0,50(0,35-0,72)$ \\
\hline
\end{tabular}

S: sensibilidad; E: especificidad; IC: intervalo de confianza; J: índice de Youden; $\mathrm{RP+}+$-: razón de probabilidad positiva/negativa; G: gastroenterólogo; E: enfermera; $\mathrm{R}$ : residente.

\section{Interpretación de los hallazgos}

La interpretación de los hallazgos por consenso y participantes se muestra en la tabla VI.

\section{Carácter de las exploraciones}

Tanto participantes como el consenso clasificaron los mismos 19 procedimientos como "anormales". No existieron discrepancias en esta variable, por lo que el acuerdo consenso-participantes fue absoluto $(\kappa=1)$.

\section{Relación entre hallazgos e indicaciones}

No se observaron diferencias significativas entre consenso y participantes cuando la relación entre los hallazgos y las indicaciones de los procedimientos se clasificó 
Tabla VI. Interpretación de hallazgos

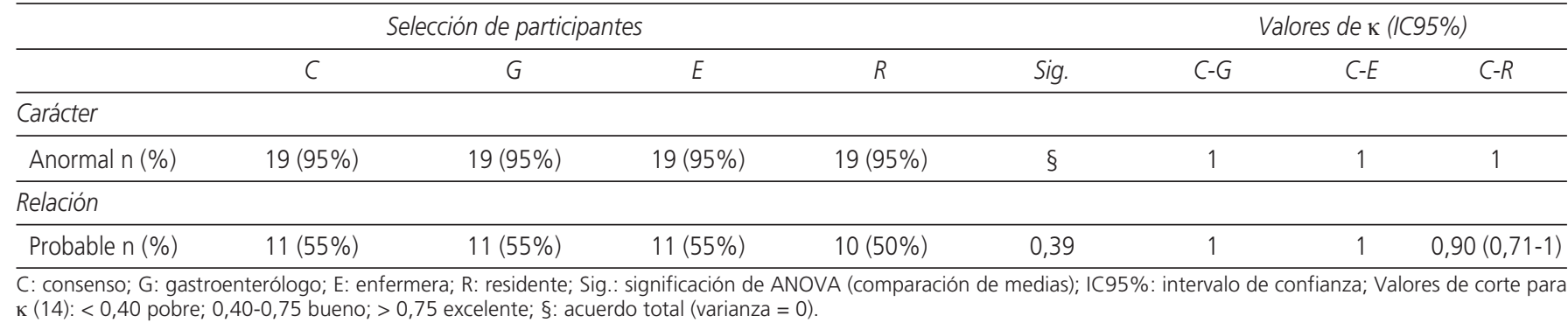

como "probable" (11/20 para gastroenterólogo y enfermera y 10/20 para el residente; $p=0,39)$. El acuerdo consenso-gastroenterólogo y consenso-enfermera fue absoluto $(\kappa=1)$ y el acuerdo consenso-residente fue excelente $(\kappa=0,90 ;$ IC $95 \%$ : 0,71-1).

\section{$t E G, t T I D$ y $t R$}

El tEG medio obtenido por el gastroenterólogo fue de $58,8 \pm 75,6$ minutos, de 58,9 $\pm 75,7$ por la enfermera y de $59,1 \pm 75,7$ por el residente $(p=0,47)$. El tTID medio obtenido por gastroenterólogo y enfermera fue de $260 \pm 84,9$ minutos y de $259,9 \pm 84,8$ por el residente $(p=0,58)$. El tiempo medio empleado en cada procedimiento fue de 51,9 $\pm 13,5$ minutos por el gastroenterólogo, $62,2 \pm 19$ por la enfermera y $60,9 \pm 17,1$ por el residente $(p<0,05)$.

\section{DISCUSIÓN}

El tiempo dedicado por el médico en la interpretación de la CE parece mayor del deseado, oscilando entre 30 y 120 minutos, según las series publicadas $(1-3,6,9)$, siendo una de las partes más costosas de este procedimiento. Alternativas al gastroenterólogo, como personal no especializado, en la interpretación de la CE no han sido profundamente analizadas. Nuestra hipótesis radica en que personal no especializado podría revisar en primer lugar las exploraciones en busca de imágenes patológicas, mientras que los gastroenterólogos podrían supervisar únicamente las imágenes seleccionadas. Esto podría significar un ahorro de tiempo y dinero. La capacidad de personal no especializado para realizar endoscopia se ha demostrado con anterioridad (15-17). Recientemente, un estudio ha evaluado por primera vez la capacidad de una enfermera de endoscopia para detectar lesiones significativas en exploraciones con CE, en comparación con un gastroenterólogo experimentado (18). Los resultados revelaron que la enfermera detectó el $93 \%$ de las lesiones significativas detectadas por el gastroenterólogo y concluyen que estas podrían revisar en primer lugar la $\mathrm{CE}$, para que el gastroenterólogo revise únicamente aquellos segmentos con patología. El principal objetivo de nuestro estudio fue evaluar la precisión diagnóstica de personal no especializado en la revisión de exploraciones con CE. Se ha evaluado además su capacidad para interpretar y caracterizar los hallazgos y el tiempo que emplean en ello. Nuestros resultados revelan que el personal no especializado selecciona más imágenes que el gastroenterólogo. Aunque su sensibilidad para el "global" de las lesiones es superior, el nivel de acuerdo con el consenso es inferior. Esto se explica por el elevado número de imágenes no patológicas que seleccionan enfermera y residente, lo que además resulta en una especificidad inferior. Como las implicaciones clínicas derivadas de perder lesiones significativas son mayores, las imágenes seleccionadas fueron clasificadas por consenso en función de su intensidad como "mayores", "no-mínimas" y "mínimas". Esto nos permitió evaluar la precisión diagnóstica de los participantes para lesiones importantes ("mayores"). La sensibilidad y la especificidad para lesiones "mayores" fueron idénticas, ya que todos detectaron las 34 lesiones "mayores" consideradas por consenso (acuerdo absoluto). Esto es de gran interés porque, en la mayoría de los casos, la detección de estas lesiones es esencial en el diagnóstico. Aunque las lesiones "no-mínimas" no son tan importantes como las lesiones "mayores", la mayoría fueron detectadas por los participantes, dando como resultado una elevada y similar sensibilidad (> 80\%). No se observaron diferencias significativas en el número de lesiones "nomínimas" detectadas por los participantes aunque el nivel de acuerdo con el consenso osciló entre pobre y bueno. Esto se debe a que enfermera y residente consideraron varias imágenes no patológicas como lesiones "no-mínimas" (falsos positivos), dando como resultado un nivel de acuerdo con el consenso inferior y una baja especificidad. Las lesiones "mínimas" fueron definidas como aquellas, a pesar de su naturaleza, inferiores a $4 \mathrm{~mm}$ en pantalla. Estas lesiones son muy frecuentes durante este tipo de exploración y habitualmente carecen de importancia diagnóstica, excepto aquellas sangrantes que se consideraron lesiones "mayores". Debido al tamaño y a su escasa importancia, parece razonable que muchas de estas lesiones pasen desapercibidas, como demuestra la sensibilidad de los participantes. En efecto, se observaron diferencias significativas en el número de lesiones "mínimas" seleccionadas por los participantes, lo que no ocurrió con las lesiones "mayores" y "no-mínimas". Como se pudo comprobar con las lesiones "no-mínimas", todos los participantes, a excepción del gastroenterólogo, incluyeron imágenes no patológicas en el grupo de las lesiones "mínimas", dando como resultado una 
pobre especificidad. Obviamente, el acuerdo consenso-participantes para estas lesiones fue pobre $(\kappa<0,45)$. Imágenes de un fotograma, pliegues polipoideos y restos de comida y/o secreciones (no se emplearon laxantes) son algunas de las imágenes clasificadas erróneamente por enfermera y residente. Se evaluó además la capacidad de enfermera y residente para caracterizar e interpretar los hallazgos. El acuerdo para la tipificación de las lesiones fue de bueno a excelente. Fue absoluto para "hemorragia activa" y excelente para lesiones "vasculares" y "erosiones-úlceras", que son las lesiones más frecuentes en la CE. Los valores de $\kappa$ para enfermera y residente, aunque aceptables, fueron ligeramente inferiores a los del gastroenterólogo. Por otro lado, la localización de las imágenes seleccionadas durante la $\mathrm{CE}$ es en ocasiones difícil para el explorador. No existen parámetros establecidos que nos faciliten esta tarea y, por ello, las referencias anatómicas juegan un papel muy importante. A pesar de que durante el entrenamiento y la lectura se emplearon criterios estandarizados para la adecuada localización de las lesiones, el nivel de acuerdo para aquellas lesiones localizadas cerca de referencias anatómicas como esófago, estómago, duodeno e íleon fue superior al resto (yeyuno). Aunque enfermera y residente obtuvieron un nivel de acuerdo de bueno a excelente, los valores de $\kappa$ fueron algo inferiores a los obtenidos por el gastroenterólogo, a excepción de la localización extraintestinal. Estos resultados demuestran que la mayoría de las lesiones importantes fueron detectadas y correctamente caracterizadas por enfermera y residente. No obstante, esto no significa que interpreten correctamente su significado en el contexto clínico. Con este propósito, se anotaron el carácter de la exploración y la relación entre las indicaciones y los hallazgos, observándose una discriminación perfecta (acuerdo absoluto) entre exploración "normal" y "anormal" y casi perfecta (acuerdo excelente) a la hora de relacionar la indicación del procedimiento con los hallazgos. Por otro lado, no se observaron diferencias significativas en el tEG y tTID medios obtenidos por los participantes, lo que significa que la selección de la primera imagen gástrica, duodenal y cecal fue muy similar. Esta selección es importante ya que es necesaria para activar el suspected blood indicator (SBI) y el sistema localizador de la cápsula. El SBI es particularmente útil en la detección de lesiones sangrantes y el sistema de localización es útil para conocer si las lesiones están al alcance de una enteroscopia convencional o para planear una enteroscopia de doble balón u otras intervenciones derivadas. Finalmente, el tiempo empleado por los participantes en cada procedimiento fue comparado y se observó que enfermera y residente eran significativamente más lentos que el gastroenterólogo, lo que probablemente se debe a que detectan más lesiones. En resumen, estos resultados demuestran que enfermera y residente, aunque más lentos, pueden detectar, caracterizar e interpretar la mayoría de las lesiones importantes durante una CE. Su elevada sensibilidad para detectar lesiones les convierte en un complemento perfecto para el gastroenterólogo en la lectura de la CE. No obstante, los gastroenterólogos deben supervisar sus hallazgos. Futuros análisis coste-efectividad nos indicarán los posibles beneficios de esta alternativa.

\section{BIBLIOGRAFÍA}

1. Ell C, Remke S, May A, Helou L, Henrich R, Mayer G. The first prospective controlled trial comparing wireless capsule endoscopy with push enteroscopy in chronic gastrointestinal bleeding. Endoscopy 2002; 34: 685-9.

2. Lewis BS, Swain P. Capsule endoscopy in the evaluation of patients with suspected small intestinal bleeding: Results of a pilot study. Gastrointest Endosc 2002; 56: 349-53.

3. Mylonaki M, Fritscher-Ravens A, Swain P. Wireless capsule endoscopy: A comparison with push enteroscopy in patients with gastroscopy and colonoscopy negative gastrointestinal bleeding. Gut 2003; 52: 1122-6.

4. Saurin JC, Delvaux M, Gaudin JL, Fassler I, Villarejo J, Vahedi K, et al. Diagnostic value of endoscopic capsule in patients with obscure digestive bleeding: Blinded comparison with video push-enteroscopy. Endoscopy 2003; 35; 576-84.

5. Hartmann D, Schilling D, Bolz G, Hahne M, Jakobs R, Siegel E, et al. Capsule endoscopy versus push enteroscopy in patients with occult gastrointestinal bleeding. Z Gastroenterol 2003; 41: 377-82.

6. Eliakim R, Fischer D, Suissa A, Yassin K, Katz D, Guttman N, et al. Wireless capsule video endoscopy is a superior diagnostic tool in comparison to barium follow-through and computed tomography in patients with suspected Crohn's disease. Eur J Gastroenterol Hepatol 2003; 15: 363-7.

7. de Franchis R, Rondonotti E, Abbiati C, Beccari G, Merighi A, Pinna A, et al. Capsule enteroscopy in small bowel transplantation. Dig Liver Dis 2003; 35: 728-31.

8. Appleyard M, Fireman Z, Glukhovsky A, Jacob H, Shreiver R, Kadirkamanathan $\mathrm{S}$, et al. A randomized trial comparing wireless capsule endoscopy with push enteroscopy for the detection of small-bowel lesions. Gastroenterology 2000; 119: 1431-8.

9. Costamagna G, Shah SK, Riccioni ME, Foschia F, Mutignani M, Perri $\mathrm{V}$, et al. A prospective trial comparing small bowel radiographs and video capsule endoscopy for suspected small bowel disease. Gastroenterology 2002; 123: 999-1005.

10. Liangpunsakul S, Chadalawada V, Rex DK, Maglinte D, Lappas J. Wireless capsule endoscopy detects small bowel ulcers in patients with normal results from state of the art enteroclysis. Am J Gastroenterol 2003; 98: 1295-8

11. Faigel DO, Baron TH, Adler DG, Davila RE, Egan J, Hirota WK, et al. ASGE guideline: Guidelines for credentialing and granting privileges for capsule endoscopy. Gastrointest Endosc 2005; 61: 503-5.

12. Halpern M, Jacob H. Atlas of Capsule Endoscopy. $1^{\text {st }}$ ed. Given Imaging Ltd.; 2002.

13. Korman LY. Standard terminology for capsule endoscopy. Gastrointest Endoscopy Clin N Am 2004; 14: 33-41.

14. Fleiss JL. Statistical methods for rates and proportions. $2^{\text {nd }}$ ed. New York: John Wiley \& Sons; 1981. p. 225-332.

15. Schoenfeld P, Lipscomb S, Crook J, Domínguez J, Butler J, Holmes L, et al. Accuracy of polyp detection by gastroenterologists and nurse endoscopists during flexible sigmoidoscopy: A randomized trial. Gastroenterology 1999; 117: 312-8.

16. Cash B, Schoenfeld P, Ransohoff D. Licensure, training, and utilization of paramedical personnel to perform screening flexible sigmoidoscopy. Gastrointest Endosc 1999; 49: 163-9.

17. Wildi SM, Wallace MB, Glenn TF, Mokhashi MS, Kim CY, Hawes RH. Accuracy of esophagoscopy performed by a non-physician endoscopist with a 4-mm diameter battery-powered endoscope. Gastrointest Endosc 2003; 57: 305-10.

18. Levinthal GN, Burke CA, Santisi JM. The accuracy of an endoscopy nurse in interpreting capsule endoscopy. Am J Gastroenterol 2003; 98: 2669-71. 